\title{
MAGNETOGRAPHIC AND SPECTROGRAPHIC OBSERVATIONS OF WEAKLY ACTIVE REGIONS
}

\author{
C. J. DURRANT
}

Cambridge Observatories, Cambridge, England

\begin{abstract}
The value of high resolution photo-electric observations of plage fields and their photospheric effects is demonstrated, with especial regard to obtaining an empirical model of a weakly active region.
\end{abstract}

\section{Introduction}

Observations of the photosphere and low chromosphere allow us to probe the structure of active regions with the minimum of difficulties arising from complex geometrical structure and non-LTE effects.

This is a preliminary report of Cambridge work on photospheric line central intensities in plage regions.

\section{Observations}

The lines observed (Table I) are all in the region of $5200 \AA$ and are mostly iron lines. They were chosen to include lines of widely different atomic characteristics. I shall

7 MAY $1970 \quad$ MCMath Plage 720

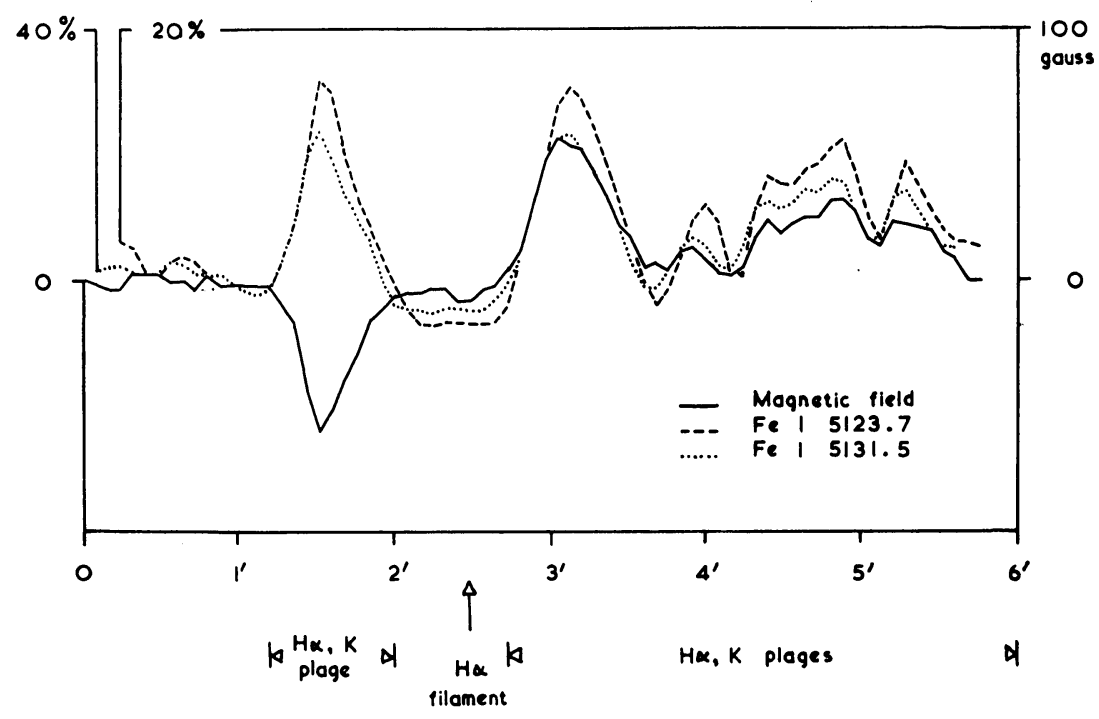

Fig. 1. Sample scan of 6 arc min of the solar disc showing longitudinal magnetic field strengths and the percentage variation of the residual intensities of the FeI lines at each point. Also indicated are the extents of visual features of $\mathrm{H} \alpha$ and $\mathrm{K}$ spectroheliograms. 
華

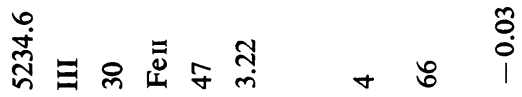

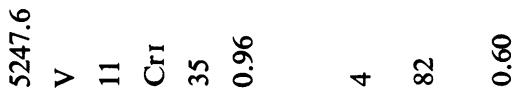

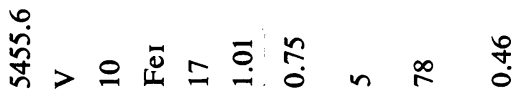

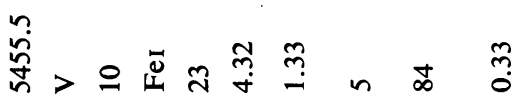

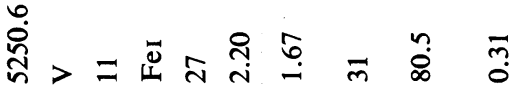

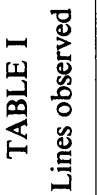

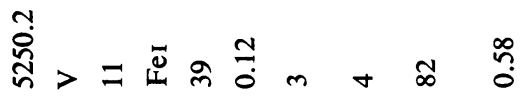

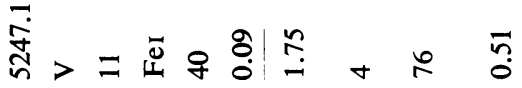

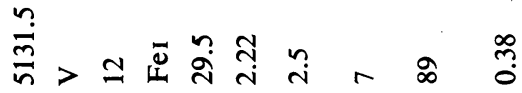

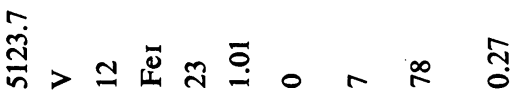

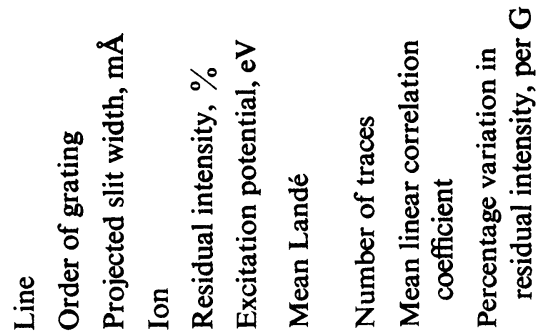


discuss mainly the observations in the $V$ order where the slit is equal to a magnetic splitting of about $500 \mathrm{G}$ or a doppler shift of $1 \mathrm{~km} \mathrm{~s}^{-1}$.

The magnetograph has an entrance slit of $5^{\prime \prime} \times 4^{\prime \prime}$ and the spectrograph slit is $5^{\prime \prime} \times 0.4^{\prime \prime}$. The line observations are smoothed so both sets of observations refer to the same effective area on the solar disc. The magnetograph employs the Fe I 5250.2 line. The magnetic and intensity records are obtained within about $3 \mathrm{~min}$ of one another. Such a set of records is shown in Figure 1 which shows the field record and simulta-

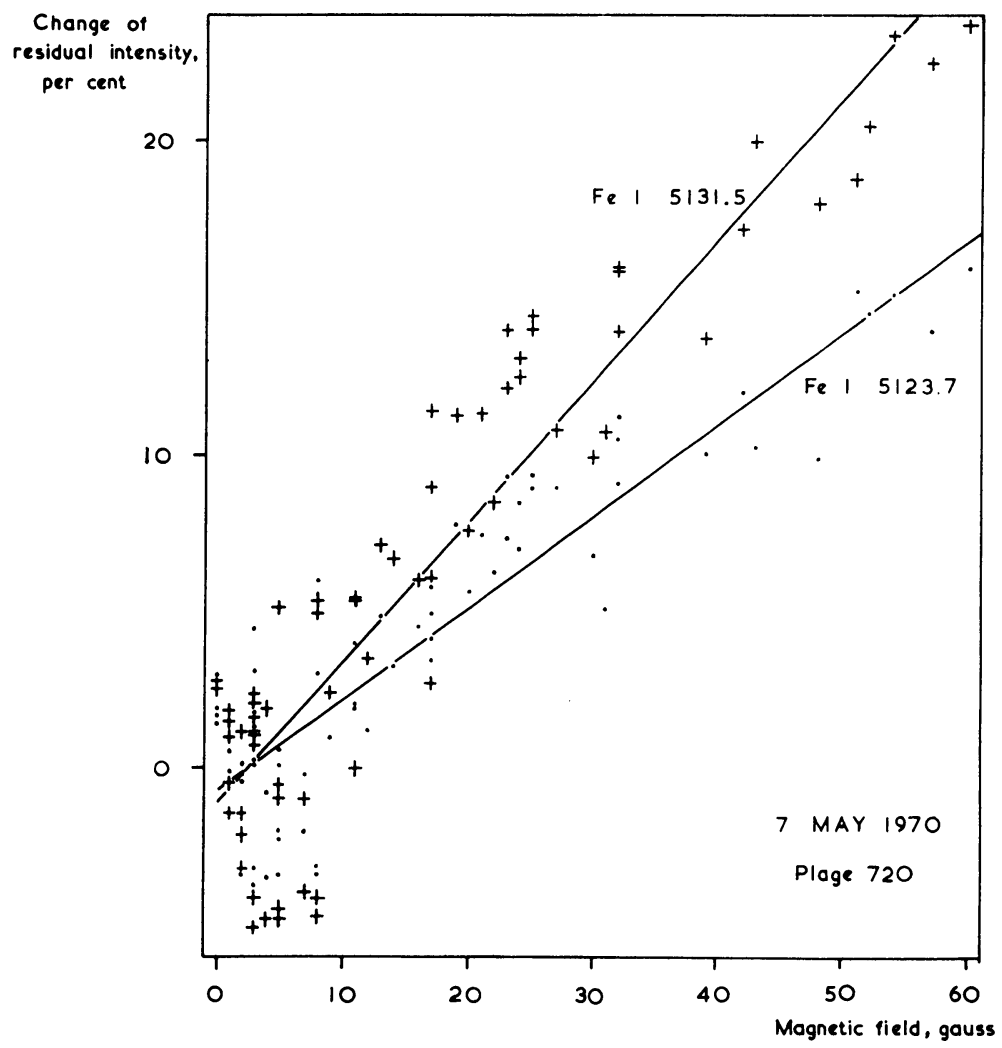

Fig. 2. The data from Figure 1 plotted to display the dependence of the percentage change of the residual intensities of the FeI lines on the longitudinal field strength. The two lines were obtained by least squares fitting.

neous records of the FeI lines 5123.7 (a magnetically null line) and 5131.5 (a Zeeman simple triplet). Typically the residual intensities of neutral ions change by $6 \%$ of continuum intensity for typical plage fields strengths which von Klüber has been careful always to describe as effective fields. It can be seen that the line variations follow the longitudinal field extremely closely for all field strengths.

Figure 2 shows a plot of percentage change of residual intensity against magnetic field strength and reveals a roughly linear relation, which is the same for fields of both 
polarities. The slope of the relation can vary markedly from plage to plage and sometimes within the same plage, by a factor of two. This must be borne in mind when averaging linear correlation coefficients and linear regression coefficients so that the 'average' behavior of the various lines in plage regions may be compared.

Table I gives the mean slope (percentage variation in line centre intensity per gauss) weighted according to the linear correlation coefficient found for each trace for each line studied. Each trace represents at least 60 independent samples. More accurate slopes will be given when more traces have been measured and prepared for computer reduction.

\section{Interpretation}

Whilst many factors can affect line centre intensities, the observations show that temperature must be the dominant factor. The FeI 5242.5 and FeII 5234.6 lines observed in the III order are formed in similar regions of the atmosphere and would show the effects of velocity fields, both micro- and macro-, with similar magnitude and sense. However this is not observed. Whereas the FeI line weakens in plage regions the Fe II line in 3 out of 4 statistically significant cases strengthened by a small

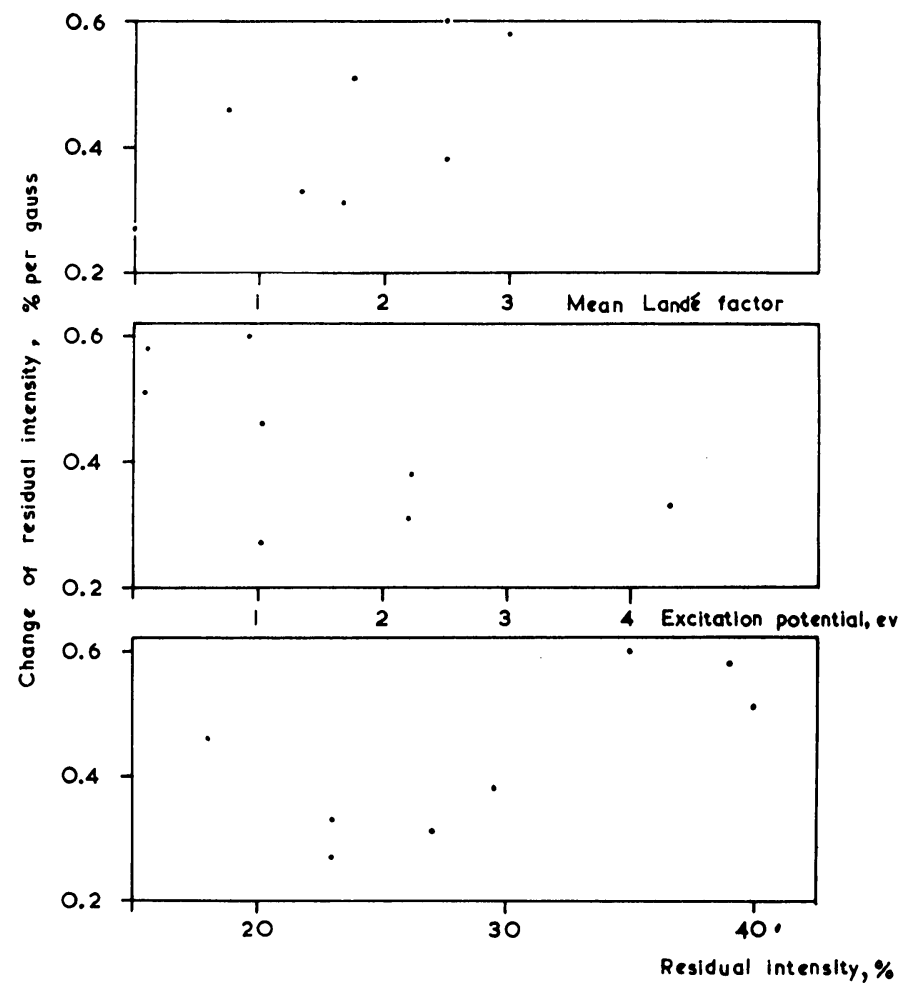

Fig. 3. Plots showing the dependence of the percentage change of residual intensity, per gauss, of the lines observed in the $V$ th order (Table $I$ ) on mean Landé factor, excitation potential and undisturbed residual intensity. 
amount. Further, the magnetically null FeI line, 5123.7, shows the same intensity variations as the other Fer lines.

The Figure 3 shows the dependence of the slope on various line parameters. There is no strict dependence on mean magnetic splitting, but there is a suggestion of a dependence on excitation potential and on central intensity, i.e. height of formation in the atmosphere.

We are computing models in hydrostatic equilibrium using a temperature structure which is perturbed from the quiet structure. Preliminary trials suggest that the observations cannot be fitted by a temperature perturbation which monotonically decreases with depth. Crude estimates, neglecting saturation and magnetic effects, suggest temperature increases of $100 \mathrm{~K}$ at $\log \tau_{5000} \sim-0.7$ and less than $50^{\circ}$ at $\log$ $\tau_{5000}<-1.0$.

We cannot insist on this conclusion until the fuller calculations are complete.

\section{Theory}

The solar atmosphere is seen to be heated in active regions by $10-100 \mathrm{~K}$ at optical depths of $10^{-1}$ to $10^{-2}$. The source of this heating is strictly correlated with effective field strength and the accepted source is dissipative processes within shock fronts. Chromospheric effects arise from magnetohydrodynamic shocks, but MHD effects will be present at optical depths of $10^{-1}$ to $10^{-2}$ only if the ratio of magnetic to gas pressure is greater than unity. This requires fields of $800-1200 \mathrm{G}$. If such fields were present, they would reduce the dissipation which is inversely proportional to the phase velocity of the wave mode carrying most of the energy. Then a vastly increased energy flux in the form of waves would be required to counter this effect and to produce the observed temperature rise.

It seems more plausible that the heating in the photospheric regions is due to ordinary hydrodynamic shocks as is that in quiet regions. Clearly the flux must be increased to account for the extra heating which occurs through earlier development of the shock front and by increased dissipation within the shock. Calculations by Michalitsanos confirm that temperature increases of the right order of magnitude can be obtained by increasing the flux by a factor of 10 in active regions with fields of the order of $100-200 \mathrm{G}$. This is in good agreement with the MHD flux required in the chromosphere.

\section{Acknowledgements}

This work was carried out jointly with Dr H. von Klüber, Mr D. W. Beggs, Miss H. A. Couper, Miss J. V. Field and Mr A. G. Michalitsanos, and was supported by the Science Research Council.

\section{Discussion}

Wiehr: Did you take care for the variations of the line depression due to the splitting? I think, realistic variations of central line depressions are only given for the non split line Fe 5123.7. Further- 
more, in my opinion there was a correlation in your last slide between the Lande factor and variation of the line depression.

Durrant: I emphasized that the temperatures I mentioned were preliminary order-of-magnitude estimates. This question can only be answered by complete computations now in progress.

Sheeley: At Kitt Peak Dr Gary Chapman and I worked on this problem. We found that the relative effect of Zeeman sensitivity and temperature on line intensity depends on the line used. As a rule-of-thumb the effect is $50-50$ for 5250 .

Pasachoff: With regard to your graph of the correlation between line intensity and magnetic field, could you please assess the errors in each of those quantities. Also, in what direction did you fit the regression line, i.e. from which quantity onto the other.

Durrant: The intensity variations were fitted to the magnetographic observations as the errors in intensity are larger.

Pasachoff: In a case like this, when both quantities are subject to errors of measurement, the leastsquares method of computing a regression line is not valid.

Durrant: I agree, but fitting a regression line by eye yields relations which are not significantly different. 